\title{
Investigation into the Role of Pump to Signal Power Ratio in FWM-based Phase Preserving Amplitude Regeneration
}

\author{
K.R.H. Bottrill*, G. Hesketh, F. Parmigiani, D.J. Richardson, P. Petropoulos \\ Optoelectronics Research Centre, University of Southampton, SO17 1BJ, UK \\ *krhb1g12@soton.ac.uk
}

\begin{abstract}
We carry out a detailed experimental characterization of a four-wave mixing based amplitude limiter in highly nonlinear fiber based on the Bessel-like power transfer characteristics and highlight trade-offs for phase preserving capabilities.

OCIS codes: (060.1155) All-optical networks; (060.4370) Nonlinear optics, fibers; (200.6015) Signal regeneration
\end{abstract}

\section{Introduction}

There is continued effort to develop all-optical phase and amplitude regenerating schemes to improve the reach of complex modulation formats in fiber optic networks, which are emerging as spectrally efficient alternatives to on-off keying. To date, much work has focused on the suppression of amplitude noise as a significant origin of phase noise in transmission due to fiber nonlinearity [1]. These schemes (which are usually phase preserving to avoid the conversion of amplitude to phase noise) have been mainly demonstrated using cross-phase modulation in a nonlinear amplifying loop mirror [2] or using saturation of four-wave mixing (FWM) interactions caused by pump depletion [3-4] in a nonlinear medium, which is usually a highly nonlinear fiber (HNLF).

A simple pump-signal FWM system may be operated as an amplitude limiter by ensuring a signal power comparable to that of the pump, resulting in pump depletion. Although it has been shown [4] that such a system need not be operated with high signal and pump power (allowing for the reduction of SPM induced amplitude to phase noise conversion, albeit with little or no signal gain), simply reducing total power may not overcome a potential cause of amplitude to phase noise conversion that we identify from the mathematical description of harmonic evolution, as resulting from the mixing of two orthogonal Bessel functions [5]. After experimentally confirming the validity of the model, we show that suppression of SPM and one of these Bessel terms, through maximization of the pump to signal power ratio, minimizes the conversion of amplitude to phase noise. We apply the system to a QPSK signal, presenting noise measurements and constellations.

\section{Background}

For the special case of a lossless and dispersionless waveguide, the nonlinear Schrödinger equation may be solved to yield $A(z, t)=A(z=0, t) e^{i \gamma|A(0, t)|^{2} z}$, where $\gamma$ is the nonlinear coefficient and $A(z, t)$ is the electric field amplitude at time $t$ and position $z$ [5]. For a typical single pump amplitude limiter, if the initial electric field consists of a pump of power $P_{0}$ and phase $\phi_{0}$ and a signal of power $P_{1}$ and phase $\phi_{1}$ at different frequencies, it can be shown that the magnitude, $\sqrt{P_{m}^{\prime}}$, and phase, $\phi_{m}$, of the $\mathrm{m}^{\text {th }}$ harmonic generated by FWM is given by [5]:

$$
\sqrt{P_{m}^{\prime}} e^{i\left(\phi_{m}^{\prime}\right)} \propto e^{i \gamma z\left(P_{0}+P_{1}\right)} \cdot\left\{i^{|m|} \sqrt{P_{0}} J_{m}\left(2 \sqrt{P_{0} P_{1}} \gamma z\right)+i^{|m-1|} \sqrt{P_{1}} J_{m-1}\left(2 \sqrt{P_{0} P_{1}} \gamma z\right)\right\}
$$

Where the pump is labelled by $m=0$ and $J_{n}$ is the $n^{\text {th }}$ Bessel function of the first kind. Two distinct causes of phase noise can be appreciated from Eq.1, the first being SPM, $e^{i \gamma z\left(P_{0}+P_{1}\right)}$, and the second the result of the mixing of two orthogonal Bessel terms. Taking the absolute square of the above equation for the signal case $(m=1)$ :

$$
P_{1}^{\prime}=P_{0}\left[J_{1}\left(2 \sqrt{P_{0} P_{1}} \gamma z\right)\right]^{2}+P_{1}\left[J_{0}\left(2 \sqrt{P_{0} P_{1}} \gamma z\right)\right]^{2}
$$

Amplitude squeezing is achieved by operating at a peak in $P_{1}^{\prime}$. With $P_{1} \sim P_{0}$, the equation describes a common regime used to perform amplitude regeneration by saturated FWM. However, by ensuring that $P_{1} \ll P_{0}$, Eq. 2 becomes:

$$
P_{1}^{\prime}=P_{0}\left[J_{1}\left(2 \sqrt{P_{0} P_{1}} \gamma z\right)\right]^{2}
$$

The advantages of minimizing the signal power as compared to the pump power are twofold; not only is SPM reduced [4], but phase modulation from the mixing of different orders of Bessel function is also eliminated.

\section{Experimental Results}

Firstly, to demonstrate the Bessel-like FWM harmonic evolution (for $1 \leq m \leq 6$ ), a pump and signal (gated with a $1 / 8$ duty cycle to reduce SBS) of frequency $f_{0}=192.46 \mathrm{THz}$ and $f_{0}=192.42 \mathrm{THz}$, respectively, were launched 

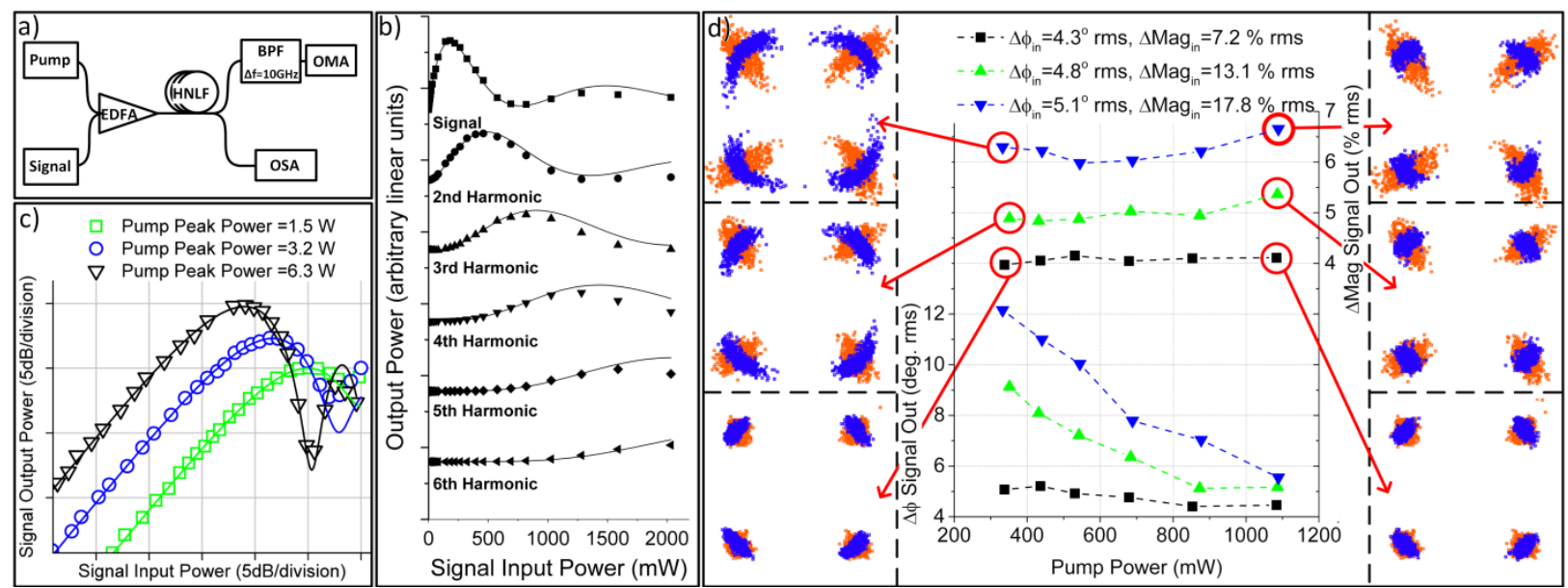

Fig. 1: a) Experimental setup. b) Measured power transfer functions of harmonics 1 to 6 for a pump peak power of $6.3 \mathrm{~W}$, fitted with their corresponding Bessel function (Eq. 1). c) Measured signal peak power transfer function for a range of pump peak powers, fitted with corresponding Bessel function in Eq. 3. d) Phase and magnitude noise of the regenerated signal against pump power for three different noise levels with accompanying constellation plots before (orange) and after (blue) regeneration.

into a cascade of strained, low dispersion HNLFs (totaling $1 \mathrm{~km}$ in length, with mean $\gamma \sim 9.5 \mathrm{~W}^{-1} \mathrm{~km}^{-1}$ ) with isolators between each segment to suppress SBS. For the experimental setup, see Fig. 1 a). Fig. 1 b) shows the output power of harmonics 1 to 6 for a pump peak-power of $6.3 \mathrm{~W}$. The data was fitted with the analytical curves (solid lines) of the respective Bessel functions from Eq. 1. The otherwise good agreement between the analytical curves and experimental data shows some divergence for higher harmonics due to dispersion effects not considered in the model. Fig. $1 \mathrm{c})$ shows the output power of the signal $(m=1)$ for different pump powers, plotted on a decibel scale. It can be seen that the signal power required to reach a peak decreases as we increase the pump power, illustrating the benefit of using high pump powers to reduce SPM in the signal. By quadrupling the pump power (from $1.5 \mathrm{~W}$ to $6.3 \mathrm{~W}$ ), the required input signal power to reach the first peak in signal output power has been reduced by $75 \%$, in accordance with Eq. 3 .

To test the ability of the scheme to preserve the phase, we used a CW pump and a 10 GBaud QPSK signal contaminated with various levels of broadband amplitude noise. Amplitude regeneration was performed on the signal using different pump powers (with the signal power being tuned to achieve optimal amplitude squeezing) and the phase noise, magnitude noise (absolute amplitude noise) and constellation plots of the signal were acquired using an optical modulation analyzer (OMA). Fig. 1 d) provides a plot of phase noise (left hand axis) and magnitude noise (right hand axis), after regeneration, against pump power (bottom axis), along with selected constellation plots. The ability of the regenerator to squeeze amplitude varies little with the pump power used, showing a $2 / 3$ reduction in magnitude noise for the case of highest noise contamination of $\Delta M a g_{\text {in }}=17.8 \% \mathrm{rms}$. On the other hand, as the pump power is increased, there is a drastic decrease in amplitude-to-phase noise conversion. For the highest pump power $(1080 \mathrm{~mW})$, there is only a marginal increase in phase noise for every noise level. In contrast, for the lowest pump power of $340 \mathrm{~mW}$ (and consequently highest signal power), in the case of highest input magnitude noise, the phase noise is increased by a factor of 3 after amplitude regeneration.

\section{Conclusion}

We experimentally confirm the Bessel-like nature of FWM harmonic evolution in a parametric amplifier and demonstrate the benefits obtained by increasing the pump to signal power ratio, substantially decreasing amplitudeto-phase noise conversion from SPM and Bessel order mixing. This approach allows phase preserving amplitude regeneration to be achieved using a single pump in a fiber optic parametric amplifier.

\section{References}

[1] J. P. Gordon and L. F. Mollenauer, "Phase noise in photonic communications systems using linear amplifiers", Opt. Lett. 15, 1351-1353 (1990).

[2] K. Cvecek, et al., "Phase-preserving amplitude regeneration for a WDM RZ-DPSK signal using a nonlinear amplifying loop mirror", Opt. Express 16, 1923-1928, (2008).

[3] K. Croussore et al., “Amplitude regeneration of RZ-DPSK signals based on four-wave mixing in fibre”, Electron. Lett., 43 (3), 177- 178, (2007).

[4] M. Matsumoto et al., "Performance improvement of DPSK signal transmission by a phase-preserving amplitude limiter", Opt. Express 15, 8094-8103 (2007).

[5] M. E. Marhic, "Fiber Optical Parametric Amplifiers, Oscillators and Related Devices", Cambridge University Press, 2007 